\title{
Podem os anti-hipertensivos estarem associados ao desenvolvimento do granuloma
}

\section{anular?}

\author{
Can anti-hypertensives drugs be associated with the development of Granuloma annulare? \\ ¿Pueden los anti-hipertensivos asociarse com el desarrollo de granuloma anular?
}

Recebido: 09/06/2021 | Revisado: 17/06/2021 | Aceito: 20/06/2021 | Publicado: 04/07/2021

Luciana Pantaleão
ORCID: https://orcid.org/0000-0001-7480-2530
Universidade Federal Fluminense, Brasil
E- mail:lupantaleao@ yahoo.com.br
Enoï Guedes Vilar
ORCID: https://orcid.org/0000-0001-8069-2104
Universidade Federal Fluminense, Brasil
E-mail: enoivilar@ @otmail.com
Miguel Augusto Martins Pereira
ORCID: https://orcid.org/0000-0001-5646-2253
Universidade Federal Fluminense, Brasil
E-mail: mappereira @icloud.com
Fundação Carlos Chagas de Amparo à Pesquisa do Esto Rido
Vitor Matheus Pinheiro Constantino
ORCID:https://orcid.org/0000-0002-5853-3892
Universidade Federal Fluminense, Brasil
E-mail: vmatpc@ gmail.com
Roger Jordan Freitas
ORCID: https://orcid.org/0000-0002-9369-283X
Universidade Federal Fluminense, Brasil
E-mail: rogerjordan6001@ gmail.com

\begin{abstract}
Resumo
O granuloma anular (GA) é uma doença inflamatória granulomatosa, considerada benigna, mas de etiologia incerta. O GA está associado a várias condições, como diabetes mellitus, malignidade, além de outras condições e medicamentos. O presente artigo é um estudo transversal, observacional e retrospectivo cujo objetivo foi analisar a associação entre os tipos de GA e os medicamentos anti-hipertensivos usados pelos pacientes antes do surgimento dessa lesão. Ainda, propomos averiguar as associações com determinadas comorbidades (hipertensão, diabetes, dislipidemia, hanseníase e malignidade), bem como mapear as áreas do corpo mais acometidas. O principal resultado foi a associação estatisticamente significante entre a utilização do atenolol e o tipo clínico disseminado $(\mathrm{p}=0,024)$ e a associação do tipo clínico localizado com a losartana $(\mathrm{p}=0,0604)$. Apesar dos resultados intrigantes, são necessários estudos mais robustos sobre essas associações, a fim de estabelecer conclusões definitivas.
\end{abstract}

Palavras-chave: Malignidade; Diabetes; Dislipidemia; Dermatologia.

\begin{abstract}
Granuloma annulare (GA) is a granulomatous inflammatory disease, considered benign, but of uncertain etiology. GA is associated with several conditions, such as diabetes mellitus, malignancy, in addition to other conditions and medications. The present article is a cross-sectional, observational and retrospective study whose objective was to analyze the association between the types of AG and antihypertensive drugs used by patients before the onset of this lesion. We also propose to investigate the associations with certain comorbidities (hypertension, diabetes, dyslipidemia, leprosy and malignancy), as well as to map the most affected areas of the body. The main result was the statistically significant association between the use of atenolol and the disseminated clinical type $(\mathrm{p}=0.024)$ and the association of the localized clinical type with losartan $(\mathrm{p}=0.0604)$. Despite the intriguing results, more robust studies of these associations are needed in order to establish definitive conclusions.
\end{abstract}

Keywords: Malignancy; Diabetes; Dyslipidemia; Dermatology.

\section{Resumen}

El granuloma anular (GA) es una enfermedad inflamatoria granulomatosa, considerada benigna, pero de etiología incierta. La GA se asocia con varias afecciones, como diabetes mellitus, neoplasias malignas, además de otras afecciones y medicamentos. El presente artículo es un estudio transversal, observacional y retrospectivo cuyo objetivo fue analizar la asociación entre los tipos de GA y los fármacos antihipertensivos utilizados por los pacientes antes del inicio de esta lesión. También proponemos investigar las asociaciones con determinadas comorbilidades 
(hipertensión, diabetes, dislipidemia, lepra y neoplasias malignas), así como cartografiar las zonas más afectadas del cuerpo. El principal resultado fue la asociación estadísticamente significativa entre el uso de atenolol y el tipo clínico diseminado $(p=0,024)$ y la asociación del tipo clínico localizado con losartán $(p=0,0604)$. A pesar de los resultados intrigantes, se necesitan estudios más sólidos de estas asociaciones para establecer conclusiones definitivas.

Palabras clave: Malignidad; Diabetes; Dislipidemia; Dermatología.

\section{Introdução}

O granuloma anular (GA) é uma doença inflamatória granulomatosa (não infecciosa), uma dermatite granulomatosa, considerada benigna. O termo granuloma anular foi cunhado em 1902 pelo Dr. Radcliffe Crocker, todavia, a mesma entidade parece ter suas primeiras descrições datadas de 1895, pelo Dr. Colcett Fox como “ringed eruption” e pelo Dr. Debreuilh como “eruption circinée cronique de la main”( Little, 1908).

Séculos passaram e ainda pouco sabemos sobre essa doença, afinal ainda não existe um tratamento estabelecido como padrão-ouro para a doença e sua etiologia é incerta (Piette et al,. 2016). Dado os aspectos histológicos, acredita-se que seja uma reação de hipersensibilidade tardia mediada por células T-helper (fenótipo Th1). Essas citocinas de perfil Th1, a ativação de metaloproteinases e a abundância de mucina teriam um papel primordial na inflamação e na destruição tecidual. (Fayyazi et al,. 2000)(Mempel et al,. 2002). O GA, de maneira geral, é uma doença que afeta qualquer sexo e idade, todavia, é mais prevalente em mulheres, em uma razão de 2:1, adultos jovens (início antes de 30 anos) e crianças. Os subtipos clínicos de GA incluem: o GA localizado, GA generalizado, GA subcutâneo (ou "deep granuloma”), GA perfurante, o GA macular ou "path" granuloma e ainda as formas consideradas atípicas, tais como o GA fotossensível, GA palmar, GA disseminado popular e GA de mucosa oral (Keimig, 2015).

Essa entidade é caracterizada por necrobiose ou degradação mucinosa do colágeno, com um núcleo de colágeno dérmico degradado, cercado por um infiltrado de histiócitos em paliçada ou intersticial. O padrão em paliçada apresenta um infiltrado de histiócitos e linfócitos ao redor de uma zona de degeneração de colágeno, enquanto que o padrão intersticial há histiócitos espalhados ao redor de feixes de colágeno e vasos sanguíneos, sendo a presença de vasculite incomum em ambos os casos. (Penas et al., 1997) (Yun et al., 2009) (Chaitra et al., 2010). É importante mencionar a coloração para mucina, uma vez que sua presença é uma característica marcante do GA, sendo muitas vezes útil na distinção de outras doenças granulomatosas não infecciosas, como sarcoidose e necrobiose lipoídica (Cohen et al, 2015). Ainda, todo o subtipo de granuloma possui suas particularidades histopatológicas.

O GA já fora associado à várias condições, como: diabetes mellitus, malignidade, sarcoidose, trauma, hiperlipidemia, exposição à luz UV, artrite reumatoide, vasculites de anticorpos citoplasmáticos anti-neutrofóbicos (ANCA) 1, doenças da tireoide e infecções pelo EBV, Parvovirus B19, HIV, HBV, sífilis ou Borrelia burgdorferi. (Lukacs et al., 2015) (Piette et al., 2016) (Cohen, 1990) (Cohen, 1991) (Cohen, 1997) (Cohen, 1999) (Cohen, 2006) (Magro et al., 1996) (Felner et al., 1997). Casos raros de defeitos imunes subjacentes também foram relatados em crianças com GA. (Mitra et al., 2005). Além disso, diversos medicamentos já foram apontados como gatilhos para a doença, embora a maioria seja apenas em casos isolados. Há reportado desde TNF-alfa, inibidores do TNF-alfa (infliximabe, adalimumabe e etanercept), calcitonina intranasal, alopurinol, topiramato, talidomida, paroxetina, anti-PD1 até terapia para artrite reumatoide juvenil (Piette et al., 2015) (Charollais et al., 2017) (Heymann,2018). Como resultado, pacientes com GA de início recente, devem ter sua história medicamentosa detalhada.

Desse modo, o presente artigo analisou a associação entre os subtipos de GA e as medicações em uso pelos pacientes antes do aparecimento do quadro, muitas delas já apontadas como gatilhos pela literatura ou utilizadas no tratamento de comorbidades implicadas no risco de desenvolver a doença. Também, nos propomos a buscar associações com as doenças em si, assim como a mapear as áreas do corpo acometidas pelas lesões e analisá-las de acordo com o subtipo clínico do 
paciente.

\section{Metodologia}

O presente trabalho é uma pesquisa de estudo de casos, de delineamento observacional transversal e retrospectivo. (Pereira et al., 2018). Foi realizado a partir de registros do setor de Dermatopatologia do Hospital Universitário Antônio Pedro (HUAP-UFF). O critério de inclusão foi ter dado entrada no Serviço para investigação durante o período proposto de 10 anos e diagnóstico de granuloma anular confirmados por biópsia, além de tipo clínico especificado pela equipe de Dermatologia do hospital. Já como critério de exclusão utilizou-se como princípio a ausência de algum dos dados relevantes a pesquisa. Desse modo, 24 pacientes foram incluídos no estudo. A idade, o gênero e a descrição clínica foram coletados dos prontuários. A análise histopatológica foi feita por 3 (três) dermatopatologistas do hospital universitário. As lâminas foram coradas em HE e coloração especial para mucina.

Por meio de testes estatísticos, buscou-se uma correlação entre os tipos clínicos de GA (localizado, disseminado), com medicamentos (losartana, atenolol, sinvastatina, metformina e levotiroxina), com doenças (neoplasias malignas, hanseníase, hipertensão, diabetes mellitus tipo 2) e com fatores de risco conhecidos implicados em varias doenças, como tabagismo e dislipidemia. O nível de significância estatística estabelecido foi de 0.05 . Para as análises de variáveis categóricas utilizou-se os softwares: IBM SPSS Statistics 20 (IBM-EUA), para Windowns versão 20.0 (IBM Corporation, Armonk, NY, EUA).

\section{Resultados}

Em relação a distribuição topográfica dos tipos clínicos, foi observado que o padrão localizado apresenta lesões concentradas em membro superior, ocorrendo em 76,92\% dos casos (Tabela 1). A segunda localização mais frequente nesse tipo clínico ocorreu no membro inferior, representando 15,38\% dos casos. Lesões na cabeça ou no pescoço representou 7,69\% dos casos. Já em relação ao tipo disseminado foi observado também maior concentração de lesões em membros superiores, presente em 90,00\% dos casos. Além disso, ainda se tratando do GA disseminado, foi observado lesões em membros inferiores e no tronco, correspondendo, respectivamente $60,00 \%$ e 40,00\% (Figura 2). Em relação aos dados epidemiológicos, foi observado maior frequência no sexo feminino, tanto no tipo localizado quanto no tipo disseminado, 84,61\% e 80,00\%, respectivamente. As idades médias entre os dois tipos clínicos mostraram-se próximos, porém foi observado maior desviopadrão no tipo disseminado (Figura 1). O tipo localizado teve idade média 57,75 (50,30 - 65,2; IC95\%) enquanto que o disseminado, idade média 43,93 (27,75 - 60,05; IC95\%).

O principal resultado quanto a análise da dependência foi a associação estatisticamente significante entre a utilização do medicamento antagonista de receptor beta-1 adrenérgico, o atenolol e o tipo clínico disseminado (Tabela 2). Afinal, todos os pacientes em uso de atenolol apresentavam esse tipo clínico, $p=0,0240$. Um outro resultado relevante foi a maior presença de tipo clínico localizado nos pacientes que faziam uso de um antagonista do receptor de angiotensina, a losartana, 75,00\% e $\mathrm{p}=0,0604$. Esses resultados tornam-se ainda mais curiosos a medida que não foi observada correlação entre nenhum tipo clinico e a hipertensão arterial sistêmica (HAS). Dentro do estudo das comorbidades, foi observado que os pacientes com DM2 apresentavam com mais frequência o tipo clínico localizado, 80,00\% dos pacientes e $p=0,339$. Não foram encontradas correlações estatisticamente significantes entre os tipos clínicos e outros medicamentos como sinvastatina, metformina, levotiroxina ou mesmo com HAS, neoplasia maligna, hanseníase dislipidemia e tabagismo. Para as análises da dependência estatística entre as variáveis categóricas foi realizado o Teste Exato de Fisher, tomando como nível de significância estatística de 0,05 . 
Tabela 1. Análise do tipo de granuloma anular quanto ao local de acometimento, membros acometidos, idade e sexo.

\begin{tabular}{|c|c|c|c|}
\hline & $\begin{array}{c}\text { Localizado, } 13 \\
(100,00 \%)\end{array}$ & $\begin{array}{c}\text { Disseminado, } 10 \\
(100,00 \%)\end{array}$ & $\begin{array}{c}\text { Perfurante, } 1 \\
(100,00 \%)\end{array}$ \\
\hline \multicolumn{4}{|l|}{ Local de acometimento } \\
\hline CAP & $1(7,69)$ & $0(0,00)$ & $0(0,00)$ \\
\hline MMS & $10(76,92)$ & $9(90,00)$ & $1(1,00)$ \\
\hline MMI & $2(15,38)$ & $6(60,00)$ & $1(1,00)$ \\
\hline $\mathrm{TCO}$ & $0(0,00)$ & $4(40,0)$ & $0(0,00)$ \\
\hline \multicolumn{4}{|l|}{$\begin{array}{l}\text { Acometimento Membros } \\
\text { simultâneo }\end{array}$} \\
\hline 1 membro & $13(100,00)$ & $3(30,00)$ & $0(0,00)$ \\
\hline 2 membros & $0(0,00)$ & $5(50,00)$ & $1(1,00)$ \\
\hline 3 membros & $0(0,00)$ & $2(20,00)$ & $0(0,00)$ \\
\hline 4 membros & $0(0,00)$ & $0(0,00)$ & $0(0,00)$ \\
\hline Média Idade & 57,03 & 50,10 & 6 \\
\hline \multicolumn{4}{|l|}{ Faixas Etárias } \\
\hline $1<20$ anos & $0(0,00)$ & $2(20,00)$ & $1(100,00)$ \\
\hline 20 anos $\leq 1<60$ anos & $7(53,84)$ & $5(50,00)$ & $0(0,00)$ \\
\hline 60 anos $\leq 1$ & $6(46,15)$ & $3(30,00)$ & $0(0,00)$ \\
\hline \multicolumn{4}{|l|}{ Sexo } \\
\hline Masculino & $2(15,38)$ & $2(20,00)$ & $1(100,00)$ \\
\hline Feminino & $11(84,61)$ & $8(80,00)$ & $0(0,00)$ \\
\hline
\end{tabular}

Legenda: $\mathrm{CAP}=$ cabeça $\mathrm{e}$ pescoço, $\mathrm{MMS}=$ membros superiores, $\mathrm{MMI}=$ membros inferiores, $\mathrm{TCO}=$ Tronco.

Fonte: Autores (2021)

Tabela 2. Análise entre a associação dos tipos clínicos de GA e os fármacos utilizados pelos pacientes antes do aparecimento dos sinais / sintomas.

\begin{tabular}{cccc}
\hline & \multicolumn{2}{c}{ Tipo Clínico } & p-valor \\
\cline { 2 - 3 } Fármacos, $n(\%)$ & Localizado & Disseminado & \\
\hline Atenolol, $4(100,00)$ & $0(0,00)$ & $4(100,00)$ & 0,024 \\
Losartana, 4(100,00) & $3(75,00)$ & $1(25,00)$ & 0,604 \\
Simvastatina, 4(100,00) & $2(50,00)$ & $2(50,00)$ & 1,000 \\
Metformina, 4(100,00) & $3(75,00)$ & $1(25,00)$ & 0,604 \\
Levotiroxina, 3(100,00) & $1(33,33)$ & $2(66,66)$ & 0,560 \\
\hline Comorbidades, $n(\%)$ & & & \\
\hline HAS, 12 $(100,00)$ & $5(41,66)$ & $7(58,33)$ & 0,214 \\
Dislipidemia, 5 $(100,00)$ & $3(60,00)$ & $2(40,00)$ & 1,000 \\
Tabagismo, 9 $(100,00)$ & $6(66,66)$ & $3(33,33)$ & 0,669 \\
DM2, 5(100,00) & $4(80,00)$ & $1(20,00)$ & 0,339 \\
Malignidade, 1(100,00) & $1(100,00)$ & $0(0,00)$ & 1,000 \\
Hanseniase, 2(100,00) & $2(100,00)$ & $0(0,00)$ & 0,486 \\
\hline
\end{tabular}

Legenda: HAS = hipertensão arterial sistêmica, DM2 = diabetes tipo 2 membros superiores.

Fonte: Autores (2021). 
Figura 1. Distribuição por idade dos tipos clínicos de GA localizado e disseminado.

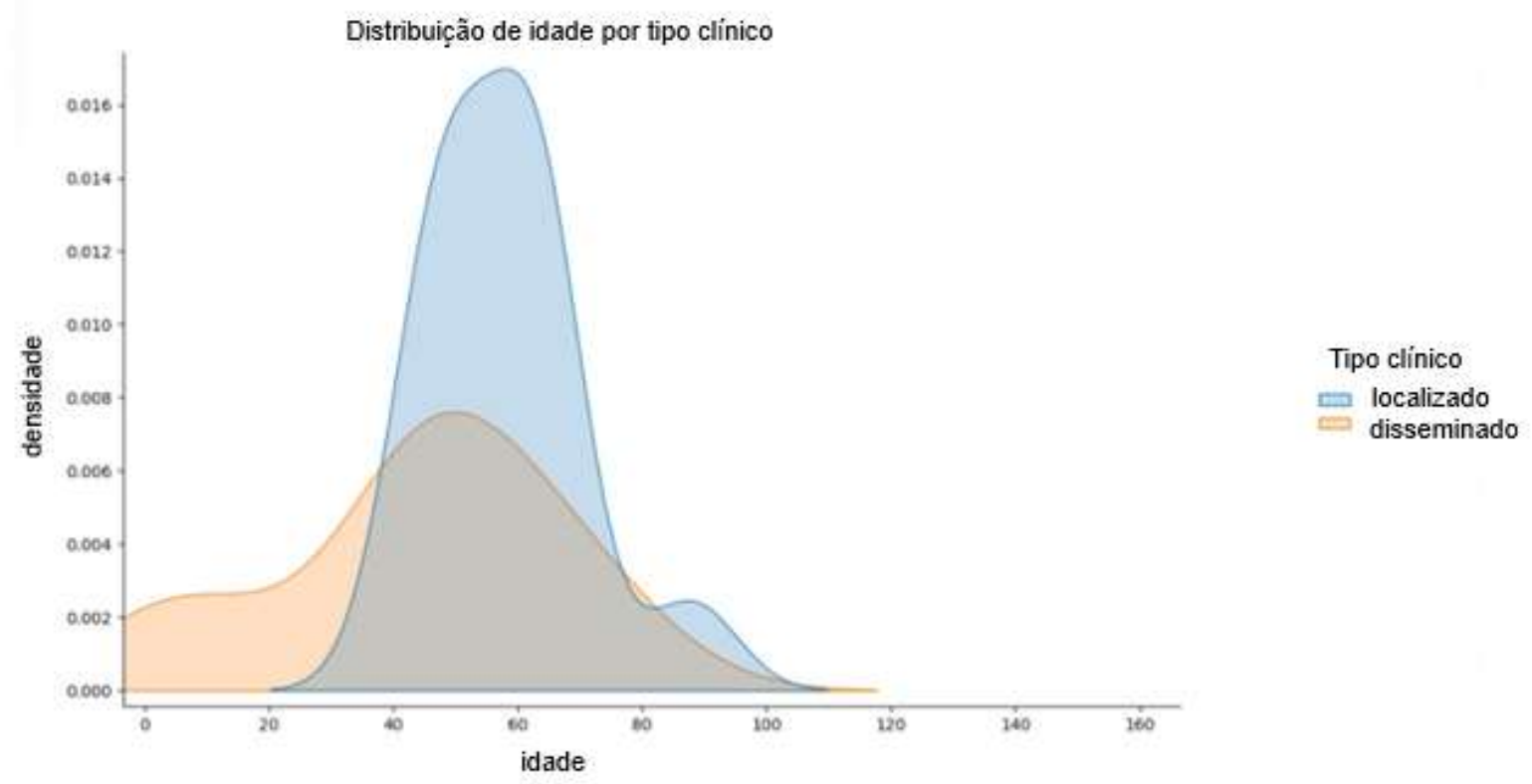

Fonte: Autores (2021).

Figura 2. (A) Granuloma anular de padrão intersticial em pequeno aumento. É possível observar infiltrado linfo-histiocitário em meio a fibras de colágeno eosinofílico (H\&E X 50). (B) Espaços entre as fibras de colágeno e os histiócitos intersticiais (H\&E X 400). (C) Fibras de colágeno eosinofílico (necrobiose) com histiócitos alongados com citoplasma claro (H\&E X 400). (D) Histiócitos intersticiais formando focalmente uma paliçada de núcleos (*) (H\&E X 400). (E e F) Paciente do sexo feminino com granuloma anular disseminado apresentando lesões em ambas as pernas.
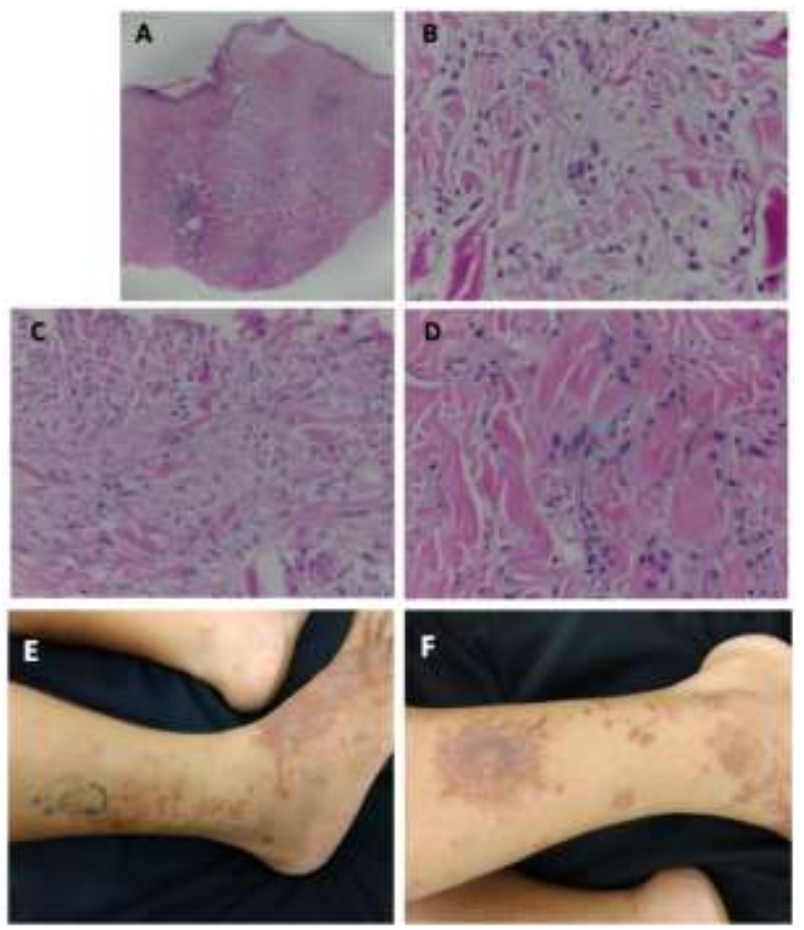

Fonte: Autores (2021). 


\section{Discussão}

O GA localizado é o subtipo mais comum, representando $75 \%$ dos casos, encontrado com mais frequência em mulheres, tende a aparecer em pacientes com idade inferior a 30 anos, e costuma se resolver dentro de 2 anos sem tratamento. (Lukacs et al., 2015) (Heymann, 2018). Nosso trabalho identificou uma concentração de pacientes com idades entre 40 e 60 anos. Esse subtipo clinico é caracterizado por pápulas anulares, cor da pele ou eritematosas, as quais crescem de maneira centrifuga e possuem menos de $5 \mathrm{~cm}$ de diâmetro e em geral acomete extremidades. Nosso estudo também apontou para preferência por extremidades, no caso, pelos membros superiores. As crianças geralmente apresentam formas localizadas e subcutâneas. Na revisão de Patrizi et al, os autores concluíram que as lesões múltiplas da variante localizada foram o subtipo mais frequente. (Patrizi et al., 1996)

Enquanto que o GA disseminado é o segundo subtipo mais comum, acometendo $15 \%$ dos pacientes (Lukacs et al., 2015), apresenta uma distribuição de idade bimodal, com picos abaixo dos 10 anos e acima dos 40, com ligeira predominância masculina. Entretanto, nossa analise mostrou incidência acentuada no sexo feminino (80\%) (Yun et al., 2009) (Patrizi et al., 2014). É definido pela presença de 10 ou mais lesões, sendo está a referência utilizada no presente artigo (Dabski et al., 1989). Acomete praticamente qualquer parte do corpo, em especial tronco, pescoço e escalpo. É o subtipo que tende a não ser autolimitado, sendo resistente aos tratamentos.

O GA subcutâneo afeta em geral crianças entre 5 e 6 anos. (Grogg et al., 2001) A GA subcutâneo se apresenta como uma massa móvel indolor, firme, em geral no couro cabeludo ou as extremidades inferiores, imitando nódulos reumatoides e resolvendo-se espontaneamente, isto é, sem intervenção cirúrgica ou medicamentosa (Pederiva et al., 2017) (Felner et al., 1997). A biópsia excecional é útil no diagnostico, mas geralmente não é curativa, com recorrências em $38 \%$ dos pacientes. (Grogg et al, 2001).

A relação do GA com o diabetes mellitus (DM) é controversa, com estudos tanto confirmando quanto refutando uma associação. (Nebesio et al., 2002) (Studer et al., 1996). Todavia, a correlação parece ser mais convincente no subtipo generalizado (Friedman-Birnbaum, 1986) (Muhlemann et al., 1984) (Mestre et al., 2014), resultado também compatível com nosso estudo. Dabski e Winkelmann, em 1989, já apontavam para uma possível associação do DM com o subtipo disseminado, ao determinar por estudo retrospectivo observacional, que $21 \%$ do grupo de referência apresentava DM, em comparação com 9,7\% dos 1350 casos de GA localizado e 10,3\% em 1383 casos de todas as formas de granuloma anular (Dabski e Winkelmann, 1989).

O trabalho de Daski e Winkelmann ainda encontrou hipercolesterolemia em 19,6\% e hipertrigliceridemia em $23,3 \%$ dos pacientes com GA. Além desse, um estudo caso-controle, publicado em 2012, apontou que $80 \%$ dos pacientes apresentavam dislipidemia, em comparação com cerca de 52\% dos controles (Wu et al., 2012). Há ainda, o relato de uma idosa japonesa com GA disseminado, com regressão após o manejo da dislipidemia (Watanabe et al., 2014). Outro estudo de delineamento caso-controle (Wu et al., 2012) examinou 140 pacientes com GA, pareando-os com 420 controles constatou dislipidemia em 79,3\% dos pacientes, em comparação com 51,9\% dos controles, com diferenças estatisticamente significativas entre os valores de colesterol total, triglicerídeos e LDL entre o grupo GA e o grupo controle. O subtipo disseminado foi o de maior prevalência de dislipidemia. Foi calculada uma razão de chances 4,04 (IC 95\%, 2,53-6,46) de pacientes com GA apresentar dislipidemia em comparação com os controles pareados. Isso nos motivou não só buscar associações com a condição (dislipidemia) em si, mas o principal medicamento para o seu manejo, a sinvastatina, porém, não foi observada correlação estatisticamente significante.

Neoplasias malignas já foram apontadas como gatilhos para o GA, sendo as hematológicas as mais relatadas, tanto linfomas Hodgkin, como não-Hodgkin e leucemias. Tumores sólidos também foram citados. Isso inclui câncer de pulmão, mama, colo do útero, cólon, próstata, testículo e tireoide (Studer et al., 1996). Nosso artigo identificou apenas 1 paciente com 
malignidade, um adenocarcinoma de pulmão, o que foi insuficiente para obter alguma correlação. Assim como a associação com DM, a relação com malignidades permanece controversa, com tanto trabalhos refutando quanto suportando (Heymann, 2018) a existência de uma correlação. Em 2019, um recente estudo caso-controle mostrou que virtualmente não há correlação entre GA disseminado e câncer, e ainda ressalta que a média de idade dos pacientes com a forma disseminado e câncer foi de 74 anos, suportando a hipótese de que a coexistência seja devido a idade avançada, como já criticaram outros autores. (Mestre et al., 2014) (Gabaldon et al., 2019)

\section{Conclusão}

Em suma, o nosso estudo constatou uma associação entre o uso de determinadas medicações anti-hipertensivas e o tipo clínico do granuloma anular, sendo o uso de atenolol e losartana associados ao aparecimento, respectivamente, dos tipos disseminado e localizado. Isso, na ausência de correlação entre esses tipos clinicos e a presença de hipertensão arterial sistêmica, porém, o estudo possui limitações em relação ao tamanho amostral e o "viés de perda". Por fim, nosso trabalho reacende uma antiga discussão da associação entre o desenvolvimento de granuloma anular e o uso de anti-hipertensivos. Todavia, indicamos a necessidade de estudos mais robustos e com maior espaço amostral, sobre essas associações, a fim de estabelecer conclusões definitivas e quiçá possíveis condutas.

\section{Referências}

Chaitra, V., Inchara, Y. K., Rajalakshmi, T. \& Antony, M. (2010). Granuloma annulare-histology reconsidered. Indian J Dermatol Venereol Leprol. 76(5), 568-9. 10.4103/0378-6323. 69050

Charollais, R., Aubin, F. \& Roche-Kubler, B. (2018). Two cases of granuloma annulare under anti-PD1 therapy. Ann Dermatol Venereol 145(2), 116-119. 10.1016

Cohen, P. R., Carlos, C. A. (2015). Granuloma annulare mimicking sarcoidosis: report of patient with localized granuloma annulare whose skin lesions show 3 clinical morphologies and 2 histology patterns. Am J Dermatopathol. 37(7), 547-50. 10.1097

Cohen, P. R., Grossman, M. E., Silvers, D. N. \& Deleo, V. A. (1990) Generalized granuloma annulare located on sun-exposed areas in a human immunodeficiency virus-seropositive man with ultraviolet B photosensitivity. Arch Dermatol. 126(6), 830-1.

Cohen, P. R., Grossman, M. E., Silvers, D. N. \& DeLeo, V. A. (1991) Human immunodeficiency virus-associated granuloma annulare. Int J STD AIDS. 2(3), 168-71. 10.1177

Cohen, P. R. (1997) Granuloma annulare associated with malignancy. South Med J. 90(10), 1056-9.

Cohen, P. R. (2006) Granuloma annulare, relapsing polychondritis, sarcoidosis, and systemic lupus erythematosus: conditions whose dermatologic manifestations may occur as hematologic malignancy associated mucocutaneous paraneoplastic syndromes. Int J Dermatol. 45(1), 70-80. 10.1111/j.13654632.2005.02667.x

Cohen, P. R. (1999) Granuloma annulare: a mucocutaneous condition in human immunodeficiency virus-infected patients. Arch Dermatol. 135(11), 1404-7.

Dabski, K., Winkelmann, R. K. (1989) Generalized granuloma annulare: clinical and laboratory findings in 100 patients. J Am Acad Dermatol. $20(1)$, 39-47.

Fayyazi, A., Schweyer, S., Eichmeyer, B., Herms, J., Hemmerlein, B. \& Radzun HJ. (2000) Expression of IFNgamma, coexpression of TNFalpha and matrix metalloproteinases and apoptosis of T lymphocytes and macrophages in granuloma annulare. Arch Dermatol. 292(8), 384-90.

Felner, E. I., Steinberg, J. B. \& Weinberg, A. G. (1997) Subcutaneous granuloma annulare: a review of 47 cases. Pediatrics. 100(6), 965-7.

Friedman-Birnbaum, R. (1986) Generalized and localized granuloma annulare. Int J Dermatol. 25,364-366.

Gabaldon, V., Gonzalez-Vico, V. (2019) Lack of an association between generalized granuloma annulare and malignancy: A case-control study. J Am Acad Dermatol. 80(6), 1799-1800. 10.1016

Grogg, K. L., Nascimento, A. G. (2001) Subcutaneous granuloma annulare in childhood: clinicopathologic features in 34 cases. Pediatrics. $107(3)$, E42.

Heymann, W. R., (2018) Granuloma Annulare's Triangular Association with Malignancy, Journal of the American Academy of Dermatology. (2018). 10.1016

Pereira A. S. et al. (2018). Metodologia da pesquisa científica. UFSM.

Keimig, E. (2015) Granuloma Annulare. Dermatol Clin. 315-329. 10.1016

Little, E. G. (1908) Granuloma annulare. Proc R Soc Med. 1(Dermatol Sect), 95-163. 
Research, Society and Development, v. 10, n. 8, e5310817015, 2021

(CC BY 4.0) | ISSN 2525-3409 | DOI: http://dx.doi.org/10.33448/rsd-v10i8.17015

Lukacs, J., Schliemann, S. \& Elsner, P. (2015) Treatment of generalized granuloma annulare - a systematic review. J Eur Acad Dermatol Venereol. 29(8), 1467-80. 10.1111

Magro, C. M., Crowson, A. N. \& Regauer, S. (1996) Lipidic tissue reactions as a manifestation of systemic disease. Hum Pathol, 27-50.

Mempel, M., Musette, P., Flageul, B., Schnopp, C., Remling, R. \& Gachelin, G. (2002) T-cell receptor repertoire and cytokine pattern in granuloma annulare: defining a particular type of cutaneous granulomatous inflammation. J Invest Dermatol. 118(6), 957-66. 10.1046

Mestre, T., Rodrigues, A. M. \& Cardoso, J. (2014) Disseminated granuloma annulare and hepatocellular carcinoma: association or coincidence? BMJ Case Rep. PMID: 25352383

Mitra, A., Pollock, B., Gooi, J., Darling, J. C., Boon, A. \& Newton-Bishop, J. A. (2005) Cutaneous granulomas associated with primary immunodeficiency disorders. Br J Dermatol. 153-194.

Muhlemann, M. F., Williams, D. R. R. (1984) Localized granuloma annulare is associated with insulin-dependent diabetes mellitus. Br J Dermatol. 111, 325329.

Nebesio, C. L., Lewis, C. \& Chuang, T. Y. (2002) Lack of an association between granuloma annulare and type 2 diabetes mellitus. Br J Dermatol 146(1), $122-4$.

Patrizi, A., Gurioli, C. \& Neri, I. (2014) Childhood granuloma annulare: a review. G Ital Dermatol. 149, 663-74.

Patrizi, A., Neri, I., Guerrini, V., Marini, R. \& Fiorillo L. (1996) Granuloma annulare, clinical and laboratory findings in a pediatric group of patients. Acta Derm Venereol. 76, 326-8.

Pederiva, F., Paloni, G. \& Berti I. (2017) Subcutaneous granuloma annulare: a diagnostic conundrum-learning from mistakes. Pediatr Emerg Care. 33(8), e30-1. 10.1097

Penas, P. F., Jones-Caballero, M., Fraga, J., Sanchez-Perez, J. \& Garcia-Diez, A. (1997) Perforating granuloma annulare. Int J Dermatol. 36(5), 340-8.

Piette, E. W., Rosenbach, M. (2015) Granuloma annulare: Clinical and histologic variants, epidemiology, and genetics. J Am Acad Dermatol. 75(3), 457-65. 10.1016 .

Piette, E. W., Rosenbach, M. (2016) Granuloma annulare: pathogenesis, disease associations and triggers, and therapeutic options. J Am Acad Dermatol. 75(3), 467-79. 10.1016

Studer, E. M., Calza, A. M. \& Saurat, J. H. (1996) Precipitating factors and associated diseases in 84 patients with granuloma annulare: a retrospective study. Dermatology. 193(4), 364-8.

Watanabe, S., Tanaka, M. \& Kobayashi, K. (2014) Remission of generalized erythematous granuloma annulare after improvement of hyperlipidemia and review of the Japanese literature. Dermatol Pract Concept. 4, 97-100.

Wu, W., Robinson-Bostom, L., Kokkotou, E., Jung, H. Y. \& Kroumpouzos, G. (2012) Dyslipidemia in granuloma annulare: a case-control study. Arch Dermatol. 148, 1131-1136.

Yun, J. H., Lee, J. Y., Kim, M. K., Seo, Y.J., Kim, M. H. \& Cho, K. H. (2009) Clinical and pathological features of generalized granuloma annulare with their correlation: a retrospective multicenter study in Korea. Ann Dermatol. 21(2), 113-9. 10.5021 